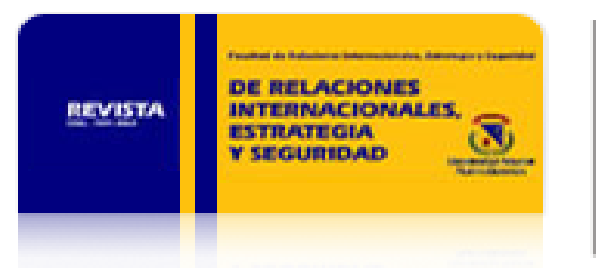

Revista de Relaciones Internacionales, Estrategia y Seguridad

ISSN: 1909-3063

cinuv.relinternal@unimilitar.edu.co

Universidad Militar Nueva Granada

Colombia

Ripoll, Alejandra

La Cooperación Internacional: Alternativa Interestatal en EI Siglo XXI

Revista de Relaciones Internacionales, Estrategia y Seguridad, vol. 2, núm. 1, enero-junio, 2007, pp.

67-83

Universidad Militar Nueva Granada

Bogotá, Colombia

Disponible en: http://www.redalyc.org/articulo.oa?id=92720104

Cómo citar el artículo

- Número completo

- Más información del artículo

Página de la revista en redalyc.org

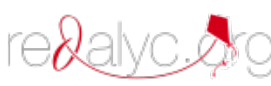

Sistema de Información Científica

Red de Revistas Científicas de América Latina, el Caribe, España y Portugal

Proyecto académico sin fines de lucro, desarrollado bajo la iniciativa de acceso abierto 
rev.relac.int.estrateg.segur.2(1):67-83,2007

\section{LA COOPERACIÓN INTERNACIONAL: ALTERNATIVA INTERESTATAL EN EL SIGLO XXI}

Por: Alejandra Ripoll ${ }^{*}$

\section{RESUMEN}

El conflicto y la cooperación son las dos interacciones básicas en las cuales se ha basado la dinámica del sistema internacional.

En las relaciones interestatales actuales, se destaca la cooperación sobre el conflicto en la solución de las controversias y para resolver los problemas mundiales que demandan la necesidad de participación multinacional.

En este sentido, una verdadera cooperación internacional en términos de corresponsabilidad, es el camino viable para una convivencia armoniosa entre las unidades del sistema internacional que cada día es más interdependiente.

\footnotetext{
* Magistra en Relaciones Internacionales, Pontificia Universidad Javeriana, profesora y editora de la Revista de la Facultad de Relaciones Internacionales, Estrategia y Seguridad de la Universidad Militar Nueva Granada. Profesora de la Facultad de Ciencia Política y Gobierno y de Relaciones Internacionales de la Universidad del Rosario. aripoll@javeriana.edu.co
} 
Palabras Clave: conflicto, cooperación, solución de controversias, problemas mundiales, interdependencia.

\section{ABSTRACT:}

The two basic interactions on which the dynamics of the international system has been based are: conflict and cooperation. Nowadays, cooperation during conflicts stands out in interestate relationships for the resolution of disagreements and to solve problems identified as worldwide.

In this sense, a true international cooperation in co responsibility terms is the viable way for a harmonious coexistence between the units of the international system, that it is every more interdependent day.

Key words: conflict, cooperation, resolution of disagreements, worldwide problems, interestate relationships

\section{INTRODUCCIÓN}

Las relaciones internacionales en el ámbito de política internacional que se ocupa de los problemas de la guerra y la paz, y de las interacciones básicas del sistema internacional como el conflicto y la cooperación, han dejado de ser un sistema de Estado para ser reemplazado por un sistema político global donde las unidades del sistema, cada día son más interdependientes. Tradicionalmente, ha existido dos formas en las cuales se relacionan los Estados: el primero, por el uso de la fuerza que en su expresión extrema es la guerra; y el segundo, la diplomacia que incluye tratados o acuerdos formales e informales que van desde la concertación, hasta su estadio superior como es la integración que contiene un alto grado de cooperación. Así, en este escrito el estudio de las relaciones políticas interestatales se tratará desde el punto del vista de la forma como se comportan los Estados en una gama amplia de asuntos donde subsiste la cooperación como eje conductor de esas relaciones, teniendo en cuenta que cada uno persigue sus metas y objetivos universales como la seguridad, el bienestar económico y social y la autodeterminación; consecución de objetivos que por supuesto, varía según su tamaño, población y recursos.

El mundo contemporáneo se caracteriza por una dependencia mutua entre los Estados, es decir, que ningún Estado puede actuar solo por muy poderoso que sea. Ejemplo de ello es el apoyo que requirió de otros ejércitos como los de España y Gran Bretaña, la superpotencia del 
mundo, Estados Unidos de América, para entrar en Irak en 2003. Así mismo, requiere de ayuda o más bien de cooperación del conjunto de los países latinoamericanos, entre otros, para solucionar o, mejor, controlar el problema de los inmigrantes y así sucesivamente, una serie retos que sin el concurso decidido de otros Estados es difícil de afrontar.

En este orden de ideas, las dos interacciones básicas en las cuales se ha basado la dinámica del sistema internacional y que se conoce también como proceso del sistema internacional, son el conflicto y la cooperación, entendiéndose por cooperación el acto por el cual se involucran dos o más actores de la misma condición que se colaboran entre sí, con el propósito de encontrar soluciones comunes que los beneficie. Por otra parte, Keohane (1988: 25), la define como "el proceso en el cual las políticas adoptadas por los gobiernos son miradas por sus contrapartes como facilitadores para lograr sus objetivos, como el resultado de coordinación de política". Así mismo, Hellen Milner lo expresa en los siguientes términos: "el comportamiento dirigido hacia una meta que implica ajustes mutuos de políticas para que todas las partes terminen mejor a que si hubiera actuado de otra manera". Estas interacciones a su vez, determinan el cambio del propio sistema internacional, la actuación de los diversos actores y la distribución de poder entre ellos.

Para entender cómo se han desarrollado históricamente las relaciones internacionales interestatales y culturales mediante los diversos actores del sistema internacional, y si en ellas ha estado presente el ánimo cooperativo, es preciso observar a las diversas maneras de comprender el mundo desde la antigüedad hasta nuestros días. Es así que en épocas pasadas, se hacía por medio de los mitos que según Cynthia Weber (2005: 6-7), es aquello que aparenta ser verdad, usualmente expresada como un lema ${ }^{1}$. En este sentido, se puede recordar la conocida obra de Maquiavelo, El Príncipe en la cual se hablaba de alianzas y de obligaciones que contraían los hombres entre sí. Así, los mitos eran las herramientas explicativas del pasado que solían dar respuestas equivalentes a las que hoy nos ofrecen las teorías científicas. Sin embargo, los mitos y las teorías no tienen un carácter determinista en la explicación de las relaciones internacionales, porque éstas las conforman hechos difíciles de explicar, y en esta medida, difícil de entender, de relacionar e interpretar.

Pese a ello, apelaremos a los paradigmas realistas y transnacionalistas, como uno de los instrumentos para probar la hipótesis con la cual parte este trabajo: la cooperación interestatal ha estado presente en todas las relaciones que se han llevado a cabo en el sistema internacional, desde la paz de Wesfalia hasta nuestros días.

\footnotetext{
${ }^{1}$ Weber cita un ejemplo de lo que es un mito en las relaciones internacionales: "internacional anarchy is the permissive cause of war" and "there is an internacional society".
} 
Este artículo consta de cuatro partes. En la primera, se examina el paradigma neorrealista a la luz del paradigma transnacionalista (institucionalismo neoliberal), como marco teórico para explicar y entender la cooperación interestatal y probar que desde la perspectiva realista, a pesar de que su juego es de suma cero, en algunos momentos la cooperación está presente, aunque de manera precaria. En la segunda parte, se revisará el concepto de cooperación internacional aunado al fenómeno de la globalización, como impulsor de la cooperación interestatal. En la tercera parte, los avances y los obstáculos que se presentan en la práctica, y por último, se plantean algunas conclusiones con miras a sintetizar los puntos relevantes de este estudio.

\section{UN MARCO EXPLICATIVO: EL REALISMO Y EL TRANSNACIONALISMO}

Cuando se habla de realismo, se descarta la cooperación como instrumento de paz. Lo mismo ocurre cuando se indaga sobre el papel de las instituciones que resulta no ser relevante para tal paradigma; por otra parte, las negociaciones no son la vía para la resolución de las diferencias, entre algunas características del pensamiento realista. Al contrario, para los pensadores realistas, la anarquía se destaca como la característica más importante del sistema internacional ${ }^{2}$, donde los Estados viven en un permanente choque, como las bolas en una mesa de billar, y el uso de la fuerza es la manera más apropiada de resolver los conflictos. Por lo tanto, reina la desconfianza entre las unidades del sistema, lo cual incide negativamente para que los Estados cooperen entre sí. Grieco (1988: 116), lo resume así: "[...] la anarquía internacional alienta la competición y el conflicto entre los Estados e inhibe sus deseos a cooperar inclusive cuando comparten intereses comunes". No obstante, los realistas tácitamente aceptan la cooperación cuando creen que los líderes nacionales "buscan maximizar las ventajas de sus países con respecto a otros Estados ya sea en cooperación con ellos o a costa de ellos"3. Pearson (2000: 158-159). Parafraseando a Morgenthau, el concepto de interés nacional no implica un mundo pacífico sino de lucha, que es posible satisfacerlo mediante un equilibrio de intereses temporal y una precaria conciliación de conflictos. En estas líneas, se puede leer que existe de una u otra manera, el acuerdo entre las unidades del sistema -así sea en un contexto histórico de guerra fría- donde los Estados estaban más preocupados por su supervivencia ante la amenaza de un ataque nuclear, y actuaban racionalmente para maximizar sus ganancias. Aún en el neorrealismo, el realismo sofisticado o realismo estructural que habla del Estado en términos de una caja negra y que destaca como principio ordenador del sistema internacional, la anarquía, éste apuesta a extender sus intereses, pero la misma naturaleza del sistema lo obliga a jugar, teniendo en cuenta los otros competidores, porque el

\footnotetext{
${ }^{2}$ Entiéndase por anarquía, la ausencia de poder central desde la perspectiva sistémica.

${ }^{3}$ Interpretación de Pearson con basada en la denominación que hizo Winston Churchill sobre interés nacional.
} 
sistema internacional constriñe a actuar a los Estados de una u otra manera. En esa medida, aunque sea difícil de mantener, reconocen que la cooperación existe ${ }^{4}$.

En esta misma dirección, se tomará la teoría de la interdependencia compleja, hoy llamada institucionalismo liberal, porque sistematiza algunos de los factores que impulsan dicho proceso. Por lo tanto, el contenido de este marco debe tomarse también, como una introducción para reflexionar sobre la importancia de la cooperación internacional como la herramienta más eficaz para el logro de los objetivos de las diferentes naciones del Mundo, mediante acciones conjuntas entre los gobiernos y demás actores de la sociedad internacional.

La teoría de la interdependencia compleja de raíces liberales, en la medida que asume que la política está fundamentalmente conformada por las fuerzas económicas, defiende las instituciones y las normas internacionales como instrumentos pacificadores y la cooperación como un juego de ganancias absolutas donde todos los que cooperan ganan. Keohane y Nye (1979), sostienen que las transnacionales, las organizaciones internacionales y otros actores del sistema internacional como las Organizaciones no Gubernamentales (ONG), nacen de la integración económica y compiten con los Estados en la escena internacional. En esta dirección indicaban con claridad que un mayor intercambio económico entre países podía ser fuente de nuevas vulnerabilidades y daría lugar, en consecuencia, a nuevas modalidades en el ejercicio del poder, pero podría abrir nuevos espacios para los países con menos poder relativo. A pesar de este planteamiento que tiene algunos tropiezos en su desarrollo, defienden la cooperación como una forma de lograr ganancias para todos ${ }^{5}$.

De igual forma, Rosenau (1996: 212), dice que: "La sociedad nacional, actualmente, se encuentra tan penetrada por el mundo exterior que ha dejado de constituir la única fuente de legitimidad, o incluso de empleo de técnicas coercitivas". Esto implica dependencia mutua que hace referencia a efectos recíprocos entre países o entre los diferentes actores del sistema internacional, además de un alto grado de influencia y participación de agentes externos en asuntos de política doméstica. Así, los efectos se presentan por las transacciones internacionales en todos los ámbitos. Sin embargo, habrá interdependencia cuando los costos de las transacciones sean recíprocos, aunque no necesariamente simétricos. De hecho, la interdependencia lleva consigo una reducción de autonomía de los involucrados y por lo tanto, implicará costos para la soberanía de un Estado; aunque los beneficios que reporta son mayores, no se puede establecer en este marco, si una relación interdependiente provocará beneficios mutuos ni necesariamente conducirá a resultados felices (Carlos Rico: 1997), porque las condiciones de los diversos Estados en una relación interdependiente no son iguales. Ejemplo de ello

\footnotetext{
${ }^{4}$ WALTZ, Kenneth N. Teoría de la Política Internacional. pp. 132-150.

${ }^{5}$ KEOHANE, Robert y NYE, Joseph. Poder e Interdependencia: La Política mundial en transición.
} 
son aquellos países que tienen mayor poder estructural, tienen mayor poder de negociación y por ende más ventajas; sin embargo, esta relación asimétrica reportará más beneficios que las relaciones conflictivas con resultado de suma cero.

Las nuevas relaciones de poder en situación de interdependencia son establecidas sobre mutuas dependencias asimétricas. Por lo tanto, deben ser entendidas en términos de sensibilidad (capacidad de respuesta), y vulnerabilidad (los actores que se ven afectados, así hagan ajustes en su política), que explican el papel de la interdependencia ${ }^{6}$. Entonces, los costos (presiones), que son impuestos a los actores pueden ser minimizados por el cambio de política, mientras que si no se logra reducirlos, las partes se encontrarán en una situación de vulnerabilidad permanente, pese a que se producirían unos procesos políticos distintos, donde los recursos de poder en poder como control de resultados, van a tener una incidencia clara para que los actores cooperen entre sí, porque el uso de la fuerza no es la herramienta adecuada para lograr acompasar los intereses de los unos y los otros en las diversas temáticas.

Por lo tanto, desde esta perspectiva, la estructura del sistema internacional ha propiciado la coordinación de políticas estatales a favor de la cooperación internacional en diversos asuntos. Esta opción de política exterior ha mostrado su capacidad para afectar procesos de tipos político y económico entre las naciones, además de la autonomía política de sus gobiernos y la dinámica de sus relaciones exteriores. También ha evidenciado su capacidad de repercutir sobre otros temas como la coordinación de programas sociales. Así, se denota claramente el surgimiento de bloques económicos, comerciales y políticos como La Unión Europea, NAFTA y Asia Pacífi$\mathrm{CO}$, que han logrado que el Mundo evolucione hacia un sistema multilateral en donde existen nuevos actores, cada uno constitutivo de un centro de poder político, económico y social.

Esta nueva coyuntura con antecedentes en el contexto de la distensión, ha originado una megacompetencia entre las naciones, pero también acuerdos que se requieren en un contexto de globalización.

\section{LA COOPERACIÓN INTERNACIONAL Y LA GLOBALIZACIÓN}

Actualmente, cuando se estudia un tema en relaciones internacionales, es necesario referirlo al fenómeno de la globalización. Según Larrahondo (2005: 42): “es una palabra de moda para abordar en la actualidad, y en cualquier nivel, temas de economía, política y finanzas, entre

\footnotetext{
${ }^{6}$ Ibid. (1979: 26-34): explican estas dos dimensiones en términos de los costos que tienen para los países en una relación interdependiente. Estos autores plantean las siguientes características de la interdependencia: el concepto pluralista, reconocimiento de actores diferentes al Estado en la escena internacional, que influyen en la toma de decisiones, una agenda multitemática y la pérdida de relevancia del uso de la fuerza en las relaciones interestatales.
} 
otros". Debido a la globalización, las transacciones comerciales y los actos de cooperación se han convertido en asuntos relevantes en las relaciones internacionales entre los diversos actores, por encima de las fronteras geográficas y la propia soberanía de los Estados. Como consecuencia de la globalización, la sociedad internacional se ha vuelto más interdependiente por una parte, mientras que por la otra, se ha producido una progresiva desigualdad entre las distintas sociedades en cuanto desarrollo, acceso a los recursos, manejo del poder entre otras cosas, que hace complejo el entendimiento de las relaciones internacionales en los actuales momentos.

En ese sentido, existen Estados poderosos que cooperan con sociedades que no tienen suficientes recursos para mejorar sus estándares de vida. Es el caso de Estados Unidos mediante la USAID ${ }^{7}$, que dispone de menos de la mitad del $1 \%$ del presupuesto federal para contribuir al desarrollo, además de la asistencia en caso de desastres, programas de salud, programas de créditos y otros. Hay rubros en los cuales, la ayuda ha aumentado, en otros, se ha mantenido y en algunos ha bajado, según las necesidades y la economía interna de los Estados Unidos. Ejemplo ${ }^{8}$ :

\section{Desarrollo de Programas de Créditos}

$\begin{array}{cccc}\text { Año fiscal } & \text { Año fiscal } & \text { Año fiscal } & \text { Requerimiento } \\ 2003 & 2004 & 2005 & 2006 \\ 7,542 & 7,953 & 7,936 & 8,000\end{array}$

Así mismo, la Unión Europea coopera con países africanos, asiáticos y de América Latina para el desarrollo. Esta ayuda oficial para el desarrollo alcanzó en 2003, 0.34\%, con proyección de $039 \%$. Lo anterior implica que existe voluntad de los países desarrollados en coadyuvar con los no desarrollados, pero no es suficiente; al contrario, en términos reales ha habido una reducción de la ayuda por varias razones, una de ellas, el déficit fiscal de los donantes, seguida de las dudas que ellos mismos tienen acerca de lo eficaz de la ayuda, como también, la pérdida de interés de la opinión pública de los países desarrollados por el proceso de desarrollo del Sur (Galán 1999: 17).

Entonces, entender la dinámica del sistema internacional hoy, demanda cada vez mayores esfuerzos "por el alto grado de interdependencia y transnacionalización [...]" (Pardo, 1995: 246). El fenómeno de la globalización y su intensificación, según Fazio “[...] está dando lugar

\footnotetext{
${ }^{7}$ Sigla en inglés de Agencia de Estados Unidos para el Desarrollo Internacional.

${ }^{8}$ http/www.USAID.gov
} 
a la emergencia de una formación social global" (Fazio, 2004: 35), en donde paradójicamente, se convierte en un factor de polarización social y económico dentro y fuera de los Estados, produciendo una nueva forma de agrupación conocida como la conformación de grandes bloques de integración materializados en tratados que fortalecen los acuerdos interestatales, transgubernamentales y transnacionales. Esto no sólo involucra a los países más desarrollados sino, "que la globalización intensa que debutó a finales de la década de los sesenta involucra de modo más directo a los países en desarrollo" (Fazio 2004: 39). Todo lo cual implica una profundización de la brecha entre los Estados ricos y los Estados pobres. En este sentido, el mundo en desarrollo precisa de más cooperación y recursos extras que provengan del mundo desarrollado con miras a robustecer sus políticas y minimizar los efectos nocivos de la globalización o como la Ilama Ulrich Beck la internacionalización del capital $^{9}$ (Beck 1995: 55-84).

Por lo tanto, la solución a los problemas del mundo en desarrollo precisa de la colaboración de los países que ostentan el poder económico, ya sea de fuentes oficiales o fuentes no gubernamentales que compartan intereses de carácter político, económico, cultural o social. En esa vía, los países pobres deben concentrar sus energías para fortalecer los lazos de cooperación entre los diversos Estados, con el propósito de enfrentar el Nuevo Orden Mundial. Entonces, es imperiosa una acción concertada basada en la confianza mutua en la esfera internacional, donde se adopten políticas coordinadas entre los diferentes organismos públicos y privados, como también en las diferentes ramas del poder público, porque "la cooperación se presenta como una herramienta estratégica para que los países del Sur puedan defenderse multilateralmente de los conceptos de "deber de injerencia y deber de asistencia", en los cuales se basan las grandes potencias para intervenir unilateralmente en los asuntos internos de los Estados" (Pardo 1995: 246). No obstante, para alcanzar una gestión concertada se requiere de un alto de grado de cooperación entre los actores del sistema internacional. La solidaridad es requisito indispensable para que la sociedad internacional avance significativamente en este propósito, asumiendo papeles de mayor participación, acción y presión, frente a las desigualdades que produce la globalización, a través de redes nacionales e internacionales que fortalezcan sus acciones; para que tengan resultados positivos como el caso de La Declaración de Doha de 2001 relativa al acuerdo sobre los $\mathrm{ADPIC}^{10}$ y la salud pública, en donde se logró reconocer los problemas graves de salud pública que afectan a países en desarrollo, especialmente el VIH/SIDA, la tuberculosis, el paludismo y otras epidemias, porque como lo expresó Ekkehart Krippendorff en su

\footnotetext{
${ }^{9} \mathrm{El}$ autor afirma que los dueños del capital han internacionalizado sus fortunas, lo cual tiene un impacto positivo para ellos y no para la humanidad. Destaca en ese sentido, la falacia de la globalización: por una parte, el aumento de la riqueza mundial, y por la otra, la disminución de la pobreza.

${ }^{10}$ Aspecto sobre propiedad.
}

LA COOPERACIÓN INTERNACIONAL: ALTERNATIVA INTERESTATAL EN EL SIGLOXXI 
obra El Sistema Internacional como Historia: "Vivimos en un mundo finito, 'interdependiente', como se dice hoy día, de pueblos obligados a estar en buenos términos entre sí, o a extinguirse juntos" (Krippendorff 1993: 9).

En este sentido, hay que tener en cuenta que la cooperación internacional como herramienta para la paz y otros asuntos concernientes al bienestar de la comunidad internacional, fue planteada en un contexto histórico de post II guerra mundial. La materialización del ánimo de cooperar de la sociedad internacional se remite a las Naciones Unidas y a las Ilamadas instituciones de Breton Woods, como el Fondo Monetario Internacional, el Banco Internacional de Reconstrucción y Desarrollo y el GATT, que si bien su objetivo principal fue establecer el Nuevo Orden Económico Mundial, éste, a su vez, implicaba el fomento del comercio internacional y la cooperación entre los pueblos. En la actualidad, estos principios han experimentado cambios profundos con el fin de la bipolaridad. Como afirma Gómez Galán "ya no necesita cumplir algunas funciones que, en cierta medida, le acompañaron desde su origen [...]" (Gómez 2001: 18).

Esos cambios han coincidido con los del sistema internacional conducentes a la formación del Nuevo Orden Mundial, lo cual exige el establecimiento de mecanismos que enfrenten al orden establecido por el imperio, que no es, como lo señala Hardt y Negri, Estados Unidos ni el imperialismo, sino aquel Estado invisible carente de los elementos del contemporáneo Estado wesfaliano conocido por todos (Hardt y Negri 2000: 44). Entonces, una de las estrategias importantes en la cual debe avanzar la cooperación internacional en un mundo interdependiente, es la firma de acuerdos, ya sean formales o informales, para que de alguna manera logren cohesionar bloques con un discurso coherente que defienda los intereses de los menos favorecidos en esta distribución de poder.

A pesar de los esfuerzos mancomunados entre Estados, organizaciones internacionales y organizaciones no estatales, enfrentar al imperio no es una tarea fácil; al contrario, es tarea de titanes porque antes de esta nueva forma de gobierno, las luchas se concentraban contra el Estadonación, mientras que hoy el imperio es descentralizado y no tiene fronteras; entonces, el nuevo imperio se funda en los organismos internacionales y desemboca en "una nueva forma global de soberanía" (Hardt y Negri 2000: ibid), además de la falta de coordinación global formal de los esfuerzos de los más débiles, que puede estar estimulando al imperio. No obstante, una identidad de intereses defendida en espacios comunes, puede ser el motor que impulse una verdadera cooperación internacional para lograr unas condiciones más justas.

Obstáculos para la Cooperación Internacional. Al fin de la guerra fría, los Estados abren la posibilidad de acciones colectivas, porque de un sistema bipolar se pasa a un sistema multipolar donde varios centros de poder compiten entre sí. Sin embargo, cabe aclarar que en recursos 
militares, los Estados Unidos no tienen competidor; en cuanto a recursos económicos existen Estados rivales, como el Grupo de los Ocho (G8), también Ilamado "el club de los ricos", conformado por Estados Unidos, Alemania, Reino Unido, Japón, Italia, Francia y Canadá; adicionalmente Rusia, que aunque ingresó en 1998, aún tiene estatus de observador.

En esta dirección, la política económica internacional tiende a la cooperación y a la integración, lo cual se traduce en una clara defensa de la cooperación sobre el conflicto, pero surge un grave problema que impide que se avance y que se hable de una verdadera cooperación en términos de corresponsabilidad y mutuas ganancias. Ese freno se refiere a las desigualdades que produce la aplicación del modelo económico actual, en circunstancias de asimetrías, refiriéndose éstas no sólo al simple poder, sino a poder estructural que incluye el económico, el tecnológico y el político, donde arroja resultados que se pueden traducir como violentos, sin el uso de la fuerza o como dice el adagio popular "golpe sin mano" a las economías en desarrollo, por los efectos nocivos que produce, como altas tasas de desempleo y desmejora de las condiciones laborales entre otros, todo esto amparado bajo el libre mercado que exige puertas abiertas sin restricciones para los poderosos, mientras que para las economías débiles, la entrada de sus productos a otras latitudes, sufren grandes restricciones como es el caso de los productos agrícolas a los mercados estadounidenses y a los de La Unión Europea, por mencionar algunos.

A pesar de esta situación, el Sistema Internacional contemporáneo ha mostrado la tendencia a propagar la cooperación internacional y a involucrar actores transnacionales, ya sean gobiernos u organismos internacionales, en la solución de los problemas domésticos o internacionales "bajo principios básicos que guarden estricta correspondencia con la génesis de los intereses comunes y problemáticas predominantes en el relacionamiento mutuo" (Garay 1998: 39). Es así como Estados Unidos, en la década de los cincuenta, contribuyó en el desarrollo económico de Taiwán, cooperando en la industria textil y de hilaturas, lo mismo que con Tailandia, en un programa de mejoramiento del arroz. Esto abre el debate sobre qué tipo de cooperación multinacional debe ser aceptada y que no viole la soberanía de los Estados, sus legislaciones internas y mucho menos los principios del derecho internacional. Pese a ello, el fenómeno de la globalización ha generado nuevas relaciones que cada día se caracterizan por ser más asimétricas en cuanto a despliegue de poder y recursos, lo cual dificulta progresar en la cooperación internacional. A pesar de que algunos países, la consideran necesaria ante las profundas desigualdades, otros no la apoyan ni se comprometen, argumentando en primera instancia que puede poner en peligro su seguridad; otro argumento que plantean es que puede afectar sus intereses económicos, y por último, algunos creen que lo más grave es que puede lesionar su soberanía.

Además, existe otro factor que influye en el no avance de una verdadera cooperación internacional, y es el desarrollo de las nuevas relaciones internacionales, con sus realidades de poder 
político y económico, y estrechos vínculos sociales y culturales, en los cuales, a veces se destaca o prima la cooperación, y a veces, se mantiene la competencia entre los diversos actores. Esto implica la reformulación teórica por una parte, para explicar esas realidades y por la otra, definir el tipo de relación entre esos actores. Sin embargo, en las diferentes agendas se evidencia que no hay opción distinta a la cooperación para enfrentar los problemas de carácter mundial que enfrenta la humanidad en pleno siglo XXI, como lo planteó Andrés Franco Vasco ${ }^{11}$ en 1995: La única opción: cooperación (Franco1995: 231). Básicamente, uno de los problemas en donde no se ha podido avanzar y es fácilmente identificable es lo relacionado con el aspecto de la seguridad. El problema se centra en que la definición de amenaza, muchas veces no es compatible con el concepto de seguridad e interés nacional de los diferentes Estados, porque "Los tipos de amenazas diferencian de manera sustantiva diversas regiones del mundo y sub-regiones dentro de ella" (Rojas 1999: 24), que dificulta el alcance de una meta común. Esto debido a que no en todas las agendas hay concordancia en lo que significa una amenaza contra sus intereses o su vulnerabilidad. Por ejemplo, para unos países, el narcotráfico puede verse como su amenaza mayor y en otros mucho menos, mientras que el terrorismo puede ubicarse en el extremo del espectro con mayor fuerza, y según esa prioridad, se diseñarían las estrategias por seguir. En este aspecto, el desarme es clave para que las unidades del sistema muestren disposición a cooperar, y en este sentido, históricamente la sociedad internacional ha intentado llegar a acuerdos como en la Conferencia de La Haya celebrada en 1899, donde se convino arreglar pacíficamente los conflictos internacionales y se trató de la reducción de armamentos y la organización jurídica de una liga internacional, y lo mismo ocurrió en el espacio de La Sociedad de Naciones. Después de la aparición de las armas nucleares en 1945, los acuerdos se suscribieron en el marco de Las Naciones Unidas, y en esa misma línea se suscribió el Tratado sobre la No Proliferación de las Armas Nucleares (TNP), vigente desde 1970. A pesar de estos intentos, la amenaza persiste hoy en día, y países como India, Pakistán e Israel que no son firmantes del TNP, aspiran a tener un gran desarrollo nuclear. Además, el retiro de Corea del Norte agrava la situación en materia de seguridad, y entonces, la salida es un desarme que se base en un compromiso negociado, donde la cooperación sea el elemento determinante entre Estados nuclearmente armados y Estados no nuclearmente armados. Como lo predijo Jesús Ruiz, en su obra El desarme en 1961: "Fórmulas no faltan [...] pero sí algo mucho más decisivo, como se ha podido comprobar también: confianza y buena voluntad mutuas" (Ruiz 1961: 79).

Algo similar ocurre en el ámbito económico, donde se requiere con urgencia la incorporación de variables que permitan visualizar los problemas desde una perspectiva global. Este inconveniente se puede subsanar en la medida en que las agendas planteen problemas comunes

\footnotetext{
11 Profesor investigador de la facultad de Ciencias Políticas de La Pontificia Universidad Javeriana. Esta sugerencia es un subtítulo del artículo Sociedad Civil y no Alineados, escrito por Franco en 1995.
} 
con soluciones comunes en un escenario de verdadera cooperación, como el Acuerdo de Comercio, Desarrollo y Cooperación (ACDC), firmado entre la Unión Europea y Sudáfrica, lo mismo que el Programa de Cooperación del Gobierno de Estados Unidos para el desarrollo de El Salvador con el USAID.

Avances para La Cooperación Internacional. A partir de la década de los noventa, se ha intentado elaborar una agenda colectiva de seguridad, partiendo de la evolución del concepto de seguridad frente a la desaparición de la amenaza del comunismo, lo cual motiva la construcción de mecanismos colectivos para enfrentar los nuevos peligros que debe enfrentar la sociedad internacional.

Desde los años setenta, en los contenidos de las agendas internacionales de los diferentes países, se refieren a la seguridad e incorporan temas de economía, medio ambiente, derechos humanos y otros, que demandan la participación multinacional para resolver los problemas comunes.

El multilateralismo ha sido exitoso en muchos campos, sobre todo en el económico, como se puede constatar con el modelo de la Unión Europea. También se ha evidenciado en la resolución de conflictos internos, cuando éstos amenazan a la estabilidad regional o a la seguridad hemisférica.

Entonces, la tendencia de los Estados hoy, es trabajar juntos para enfrentar juntos los diferentes asuntos y problemas de la agenda, debido a la interdependencia creciente. La cooperación se ha convertido en un elemento central de las relaciones interestatales entre los regímenes que son reglas y normas aceptadas por los Estados como una forma de coexistencia.

Precisamente, el mundo avanza hacia una sociedad internacional que hace énfasis en la persuasión, la negociación y la cooperación en vez del conflicto, para lograr una convivencia pacífica y de alguna manera, regular sus intereses.

Entonces, pasar de un estado hobbesiano de guerra al estado lockiano de la sociedad civil, es avanzar hacia un estado de armonía donde el uso de la fuerza se desplaza y se llega a la búsqueda de otros instrumentos como la cooperación. El hecho de pertenecer al Sistema Internacional, implica aceptar el principio de la cooperación internacional como mecanismo eficaz en la solución pacífica de las controversias y como herramienta de desarrollo. Una de las evidencias más palpable de ello, es la firma de la Carta de las Naciones Unidas en San Francisco, el 26 de junio de 1945, cuando los Estados parte se comprometieron a "realizar la cooperación internacional en la solución de problemas internacionales de carácter económi- 
co, social, cultural o humanitario, y en el desarrollo y estímulo del respeto a los derechos humanos y a las libertades fundamentales de todos, sin hacer distinción por motivos de raza, sexo, idioma o religión [...] $]^{12}$. Pese a ello, "Las Naciones Unidas y sus agencias especializadas en el desarrollo económico y social a menudo se convirtieron en escenarios del conflicto bipolar, y ello contribuyó a paralizarlas y a debilitar su actuación en este campo" (Sanahuja 2001: 67). Así, no sólo interrumpió su actuación en el campo de la cooperación, sino que poco a poco se fue desdibujando su carácter de órgano representativo de la manifestación de la voluntad de los diversos Estados. A pesar de ello, para Henderson, los organismos internacionales trascienden las fronteras, facilitan la cooperación entre los miembros, permiten reglar la conducta de los Estados, promueven la cooperación sobre el conflicto y lo más importante, contribuyen a la formación de una sociedad internacional que trasciende la anarquía de los Estados proclives al conflicto (Henderson 1998: 385), lo cual que llevaría a concluir que las organizaciones internacionales, aún pueden tener un papel preponderante en los actuales momentos que vive la sociedad y sin duda alguna, es la palabra de la diplomacia multilateral.

En este sentido, la cooperación no sólo se remite a la apertura de fronteras económicas, sino a otros ámbitos como la seguridad, los asuntos medioambientales, la tutela de los derechos humanos y el combate del crimen transnacional, entre otros. Aunque en esta búsqueda, es claro que hay distintos intereses y objetivos, además de prioridades, la sociedad internacional sigue impulsando el proceso y buscando puntos medios de entendimiento. A pesar de todas las dificultades, un avance significativo para la cooperación internacional es la consolidación de Estados democráticos, que neutraliza la tendencia que ha tenido el Sistema Internacional de tener relaciones conflictivas. Aunque las asimetrías de poder podrían desdibujar en sentido amplio, la cooperación entre los diferentes Estados, las mismas a su vez, permitirán generar mejores espacios para la negociación.

Así, la teoría de juegos que habla del cálculo costo/beneficio, nos demuestra que es mucho más rentable defender los intereses propios en un espacio común que en espacios cerrados. Por lo tanto, problemas como la pobreza, las asimetrías económicas y muchos otros que afectan gran parte de la población mundial, por su carácter de global, no se pueden tratar desde un nivel individual, sino por medio de acciones conjuntas, en las cuales se encuentre por lo menos, una identidad de intereses, porque habrá mayor tendencia a la cooperación en ambientes donde converjan intereses comunes y complementarios, y no opuestos.

\footnotetext{
${ }^{12}$ CARTA DE LAS NACIONES UNIDAS. Capítulo I Propósitos y Principios. Artículo 1. Citado por: VERGARA, Alberto. Derecho Internacional. 2 ed. Santafé de Bogotá: La Constitución Ltda., 1995. p. 442.
} 


\section{CONCLUSIÓN}

Las dos interacciones básicas en las cuales se ha basado la dinámica del sistema internacional que se conoce también como proceso, son el conflicto y la cooperación. Estas interacciones a su vez, determinan el cambio del propio sistema internacional, la actuación de los diversos actores y la distribución de poder entre ellos.

Hoy en día, se destaca la cooperación sobre el conflicto en las relaciones interestatales para la solución de las controversias, y para resolver los problemas identificados como mundiales que demanden la necesidad de participación multinacional, materializada en la firma de tratados bilaterales y multilaterales que han surgido entre Estados o grupos de Estados en los últimos años, cuando se denotan las formas y grados de cooperación.

Sin embargo, hay dificultades para que avance significativamente la cooperación internacional. Por una parte, las asimetrías de poder, tanto en lo económico, como en lo político y lo tecnológico, y por la otra, la competencia entre los actores y la dificultad de unificar criterios acerca del concepto seguridad y lo que significa una amenaza real o potencial.

Con todo, la tendencia actual de los Estados es trabajar juntos para enfrentar juntos a los diferentes asuntos y problemas de la agenda, debido a la interdependencia creciente. Entonces, la cooperación se ha convertido en un elemento central de las relaciones interestatales por medio de los regímenes que son reglas y normas aceptadas por los Estados como una forma de coexistencia

Esto ha conducido u obligado a los actores estatales y no estatales, a buscar mecanismos de entendimiento en el ámbito económico y también, a acompasar sus intereses en lo político y social, porque sólo el esfuerzo colectivo puede lograr una mejor convivencia con un impacto positivo sobre la paz mundial.

Esta conclusión más bien optimista, permite trazar un camino favorable hacia la cooperación con los países en vía de desarrollo, aunque no suficiente, desde los países desarrollados, pero en ese mismo sentido, permite sugerir algunos lineamientos para el avance en la cooperación:

Primero, es necesario construir orientadas a facilitar la cooperación al desarrollo y que abarquen un suficiente número de metas, pero no tantas que se pierdan en la intención. En este caso, se requiere identificar y priorizar las necesidades comunes para el desarrollo de los países del Sur.

LA COOPERACIÓN INTERNACIONAL: ALTERNATIVA INTERESTATAL EN EL SIGLO XXI 
Segundo, coordinar ampliamente ${ }^{13}$ proyectos conjuntos entre países pobres y países ricos, a partir de la identificación de las necesidades principales comunes.

Tercero, mediante algunas instituciones estatales, entes privados y ONG de los diferentes Estados, regular la canalización de los recursos y la organización de las tareas, con el fin de establecer una política común, dirigida al cumplimiento de objetivos predeterminados, porque los Estados suelen reaccionar en forma distinta a la ayuda externa, lo cual desvirtuaría la intención común.

Cuarto, despolitizar el andamiaje tendiente a lograr el cumplimiento de los objetivos como condición para que se dé un avance significativo en el desarrollo.

En definitiva, el proceso de cooperación no es una empresa fácil, pero sí es cierto y concluyente que por ser una identidad de intereses defendida en espacios comunes, puede ser el motor para impulsar una verdadera cooperación internacional, lograr unas condiciones más justas en los momentos actuales y una convivencia armoniosa entre las unidades del sistema internacional, en correspondencia con una estrategia común que la privilegie sobre el conflicto.

\section{BIBLIOGRAFÍA}

BECK, U. (1998). ¿Qué es la Globalización? Falacias del Globalismo, Respuestas a la Globalización. Barcelona: Paidós Ibérica.

FAZIO, H. (2004). El Mundo en los Inicios del siglo XXI. Bogotá: Universidad de los Andes.

FRANCO, A. (1995). Sociedad Civil y No alineados. Santafé de Bogotá: Revista Javeriana, pp. 229-241.

GARAY, L. J. (1998). ¿Corresponsabilidad Real o Recreación de Utopías? En: Estrategia Económica y Financiera, 269. Santafé de Bogotá, pp. 39-42.

GÓMEZ, G. M. (2001). La Cooperación al Desarrollo en un mundo en cambio. Madrid: Cideal.

GRIECO, J. (1988). Anarchy and the limits of cooperation. International organization, vol 42. New York.

\footnotetext{
${ }^{13}$ Ampliamente en este caso, implica tratados de tipo multilateral y no bilateral.
} 
HARDT, M. y NEGRI, A. (2000). Imperio. Cambridge: Harvard University Press.

HENDERSON, C. (1998). International Relations: conflict and cooperation at the turn of the 21st century. Newman: McGraw-Hill.

KEOHANE, R. y NYE, J. (1995). Poder e Interdependencia. Citado por: BARBÉ, Esther. Relaciones internacionales. Madrid: Tecnos.

KEOHANE, Robert. (1979). Power and Interdependence. Citado por: TORO, Celia. Narcotráfico: lo que la interdependencia no nos explicó (1997). En: La política Exterior de México: enfoques para su análisis. México: El Colegio de México, Instituto Matías Romero de Estudios Diplomáticos.

. (1988). Poder e interdependencia: La Política mundial en transición. Buenos Aires: Grupo Editor Latinoamericano.

. (1984). After Hegemony: Cooperation and discord in the World political economy. Princeton: Princeton University Press.

KRIPPENDORFF, E. (1993). El Sistema Internacional como Historia, Introducción a las relaciones internacionales. México: Fondo de Cultura Económica.

LARRAHONDO, M. C. (2005). Economía, Gestión y Desarrollo. Cali: Pontificia Universidad Javeriana - Facultad de Ciencias Económicas y Administrativas.

MILNER, H. (1995). Citado por: PARDO, D. La Cooperación: característica fundamental de los NOAL. Santafé de Bogotá: Revista Javeriana, pp. 243-247.

PARDO, D. (1995). Ibid.

PEARSON, F. y ROCHESTER, M. (2000). Relaciones Internaciones: situación global en el S. XXI. Bogotá: McGraw-Hill.

RICO, C. (1997). The Future of Mexican-US. Relation and the Limits of the Rhetoric of Interdependence. Citado por: Celia Toro. Op. Cit.

ROJAS A. F. (1999). Cooperación y seguridad Internacional en las Américas: ¿nuevos conceptos, nuevos riesgos, nuevas amenazas. En: Cooperación y seguridad Internacional en las Américas. FLACSO-Chile. Caracas: Nueva Sociedad. . 
ROSENAU, J. (1996). Preteoría y teorías sobre política exterior. En: VÁSQUEZ, John, editor. Las clásicas relaciones internacionales. México: Limusa.

RUÍZ, J. (1962). El Desarme. Barcelona: Ediciones G.P.

SANAHUJA, J. A. (2001). En: La Cooperación al Desarrollo en un mundo en cambio. Madrid: Cideal.

VÁSQUEZ, J. (1996). Relaciones Internacionales. El pensamiento de los Clásicos. México: Limusa.

VERGARA, A. (1995). Derecho Internacional. 2 ed. Santafé de Bogotá: La Constitución Ltda.

WALTZ, K. (1979). Teoría de la Política Internacional. Buenos Aires: Grupo Editor Latinoamericano.

WEBER, C. (2005). Internacional Relations Theory. A critical introduction. 2 ed. London- New York: Routledge. 